\title{
Empfehlungen notwendiger Kontrolluntersuchungen bei okulärer Hypertension
}

Töteberg-Harms, M ; Hirn, C ; Funk, J

\begin{abstract}
Der Begriff okuläre Hypertension(OHT) bezeichnet einen über die „Norm“ (> $21 \mathrm{mmHg}$ ) erhöhten Intraokulardruck (IOD) bei gleichzeitig offenem Kammerwinkel, ohne dass glaukomtypische Gesichtsfeldausfälle oder Papillenveränderungen vorkommen. Zudem fehlen ophthalmologische oder systemische Erkrankungen, die sekundär zu einem erhöhten IOD führen. Synonym werden die Begriffe Glaukomverdacht, primäres Offenwinkelglaukom ohne Schaden oder frühes Glaukom gebraucht. Wichtig ist, dass es sich um Individuen handelt, die ein erhöhtes Risiko haben, ein Glaukom zu entwickeln. Für ein korrektes Patientenmanagement ist es elementar, ein festes Schema zur Basisdiagnostik und v. a. zum Follow-up mit sinnvollen Kontrollintervallen zu haben.
\end{abstract}

DOI: https://doi.org/10.1007/s00347-011-2382-1

Posted at the Zurich Open Repository and Archive, University of Zurich

ZORA URL: https://doi.org/10.5167/uzh-51162

Journal Article

Published Version

Originally published at:

Töteberg-Harms, M; Hirn, C; Funk, J (2011). Empfehlungen notwendiger Kontrolluntersuchungen bei okulärer Hypertension. Der Ophthalmologe, 108(11):1016-1022.

DOI: https://doi.org/10.1007/s00347-011-2382-1 
Ophthalmologe $2011 \cdot$ 108:1016-1022

DOI 10.1007/s00347-011-2382-1

(c) Springer-Verlag 2011
M. Töteberg-Harms - C. Hirn - J. Funk

Augenklinik, UniversitätsSpital Zürich
Der Begriff okuläre Hypertension $(\mathrm{OHT})$ bezeichnet einen über die "Norm“ (>21 mmHg) erhöhten Intraokulardruck (IOD) bei gleichzeitig offenem Kammerwinkel, ohne dass glaukomtypische Gesichtsfeldausfälle oder Papillenveränderungen vorkommen. Zudem fehlen ophthalmologische oder systemische Erkrankungen, die sekundär zu einem erhöhten IOD führen. Synonym werden die Begriffe Glaukomverdacht, primäres Offenwinkelglaukom ohne Schaden oder frühes Glaukom gebraucht. Wichtig ist, dass es sich um Individuen handelt, die ein erhöhtes Risiko haben, ein Glaukom zu entwickeln. Für ein korrektes Patientenmanagement ist es elementar, ein festes Schema zur Basisdiagnostik und v. a. zum Follow-up mit sinnvollen Kontrollintervallen zu haben.

\section{Epidemiologie}

Die Kriterien der okulären Hypertension treffen auf ca. 4-10\% aller über 40-Jährigen zu [1, 9]. OHT kommt je nach Abstammung unterschiedlich häufig vor, so in $18,4 \%$ bei Schwarzafrikanern, $13,6 \%$ bei Individuen mit gemischter Abstammung und nur bei 4,6\% der Kaukasier desselben Alters [10].

\section{Konversionsrate}

Die Konversionsrate von OHT zu einem primären Offenwinkelglaukom (POWG) mit Gesichtsfeldausfällen liegt lediglich bei $0,5-1,0 \%$ pro Jahr $[7,11]$.
In die Ocular Hypertension Treatment Study (OHTS), einer randomisierten, prospektiven, klinischen Multicenterstudie, wurden von 1994 bis 1996 insgesamt 1636 Patienten eingeschlossen. Ziel war es, die Effizienz lokaler IOD-senkender Medikamente zur Verzögerung oder Verhinderung der Konversion in ein Glaukom zu bestimmen. Das Follow-up betrug im Median 72 bis 78 Monate. In der Gruppe, die lokale IOD-senkende Medikamente erhielt, entwickelten $4,4 \%$ ein Glaukom, während dies bei 9,5\% der Patienten der unbehandelten Kontrollgruppe eintrat [6].

\section{Therapiebeginn und Therapieoptionen}

Dies begründet das Dilemma, wen behandeln und wen nicht. Alle Individuen mit OHT zu behandeln scheint unangebracht, bedenkt man die geringe Konversionsrate ebenso wie Kosteneffektivität, Nebenwirkungen der Therapie und Non-Compliance. Des Weiteren hat man Zeit, bis im Fall einer Konversion eine Therapie begonnen werden muss. Erst bei einem Verlust von mehr als ca. 40\% der Axone des N. opticus kommt es zu Gesichtsfeldausfällen in der Perimetrie und somit zu einer funktionellen Einbuße. Es kann also argumentiert werden, dass es ausreichend ist, bei regelmäßiger Kontrolle eine Therapie erst bei nachgewiesener Konversion und Progression zu initiieren. Das „Für und Wider" einer Therapie bei OHT wird in gesonderten Beiträgen in dieser Ausgabe von Der Ophthalmologe ausführlich diskutiert.

\section{( Unter Kontrolle muss eine Therapie erst bei nachgewiesener Konversion begonnen werden}

Als Therapieoptionen kommen die gleichen medikamentösen Therapien in Betracht wie beim Offenwinkelglaukom (- Infobox 1).

\section{Basisdiagnostik}

Ziel der Basisuntersuchung bei OHT ist einerseits, eine allfällige, bereits vorliegende glaukomatöse Schädigung des Sehnerven oder des Gesichtsfeldes und andererseits sekundäre Ursachen für eine Erhöhung des Augendruckes auszuschließen.

Dies bedeutet, dass initial eine vollständige Glaukomdiagnostik empfehlenswert ist mit:

- Anamnese,

- Spaltlampenuntersuchung,

- Gonioskopie,

- Pachymetrie,

- Messung des Intraokulardruckes,

- Perimetrie,

\section{Infobox 1 Leitlinien zum Thema}

okuläre Hypertension

- Die Deutsche Ophthalmologische Gesellschaft (DOG) veröffentlicht auf der Homepage der Arbeitsgemeinschaft der Wissenschaftlichen Medizinischen Fachgesellschaften e.V. (AWMF), www.awmf.org, Leitlinien zum Thema okuläre Hypertension

- European Glaucoma Society (EGS):„,Terminologie und Handlungsrichtlinien für die Glaukome" (3. Auflage), www.eugs.org

- National Institute for Health and Clinical Excellence (NICE), www.nice.org.uk/cg 
Hier steht eine Anzeige.

黛 Springer 


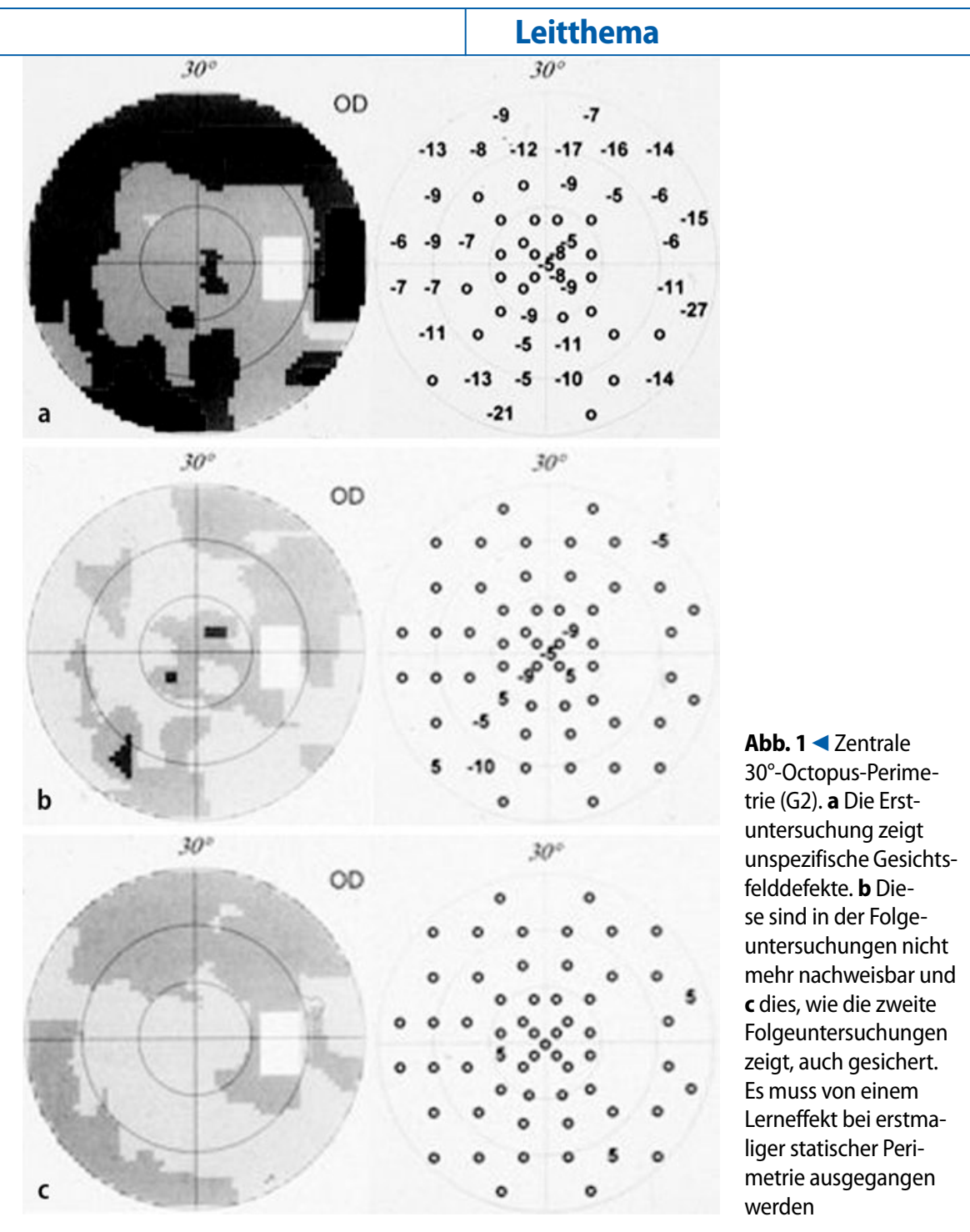

- biomikroskopischer Papillenbeurteilung,

- Papillen- und Nervenfaserdokumentation.

\section{Anamnese}

Mit der Anamnese sollen unter anderem Risikofaktoren für die Entwicklung eines Glaukoms erfasst sowie mögliche sekundäre Ursachen für eine Augendrucksteigerung aufgedeckt werden. Auf folgende Punkte sollte daher in der Anamnese besonders geachtet werden:

- Familienanamnese - sowohl allgemeinmedizinisch als auch ophthalmologisch,

- allgemeine und ophthalmologische Krankengeschichte inklusive früherer und aktueller Medikation mit Augenmerk auf Kortikosteroidmedikation,

- Contusio bulbi,
- Hornhautchirurgie (z. B. Status nach Keratoplastik, refraktiver Chirurgie, Crosslinking).

\section{Spaltlampenuntersuchung}

In der Spaltlampenuntersuchung sollen sekundäre Ursachen für eine Augendruckerhöhung ausgeschlossen werden, wie z. B. ein Pseudoexfoliations- (PEX) oder Pigmentdispersionssyndrom (PDS) und Entzündungen. Außerdem kann die thode nach van Herick abgeschätzt werden $[4,14]$. Dabei wird die periphere Vorderkammertiefe als Abstand zwischen Iris und Hornhautendothel in Relation zur Hornhautdicke beurteilt (• Tab. 1). Ab einer Vorderkammertiefe von Grad III nach van Herick ist ein Kammerwinkelverschluss als unwahrscheinlich anzusehen. Vorderkammertiefe bereits mit der Me-
Die Spaltlampenuntersuchung erlaubt auch, Veränderungen aufzudecken, welche die Ergebnisse der Applanationstonometrie verfälschen könnten, wie ein Hornhautödem oder eine Bandkeratopathie.

\section{Gonioskopie}

Bei der Gonioskopie muss in erster Linie ein offener Kammerwinkel (Shaffer [13] Grad III oder IV) bestätigt werden. Zusätzlich sollen sekundäre Ursachen für einen erhöhten IOD, wie z. B. Neovaskularisationen, Art und Grad der Pigmentierung oder Synechien, ausgeschlossen werden.

Empfehlenswert ist initial eine einmalige Untersuchung mit einem Gonioskopiekontaktglas. Andere Methoden der Vorderabschnittsdarstellung, wie z. B. Vorderabschnitts-OCT, Scheimpflug-Kamera, oder Ultraschallbiomikroskopie, sind für die routinemäßige Basisuntersuchung weniger geeignet.

\section{Pachymetrie}

Die zentrale Hornhautdicke (CCT) ist unabhängig vom Einfluss auf den IOD [12] ein wesentlicher Risikofaktor für die Progression einer OHT in ein POWG. Die Messung der CCT ist daher essenziell bei der Beurteilung einer OHT.

Die CCT kann mittels Ultraschall oder „non-contact“ z. B. mit einer Scheimpflug-Kamera gemessen werden. Dies sollte standardmäßig bei allen Basisuntersuchungen erfolgen, da eine überdurchschnittlich dicke Hornhaut dazu führt, dass v. a. mit der Applanationstonometrie fälschlich zu hohe Intraokulardrücke (IOD) gemessen werden. Bei unterdurchschnittlich dünnen Hornhäuten wird hingegen fälschlicherweise ein zu niedriger IOD applanatorisch gemessen. Versuche, Formeln zur direkten Umrechnung des applanierten IOD hin zum tatsächlichen IOD, wie er bei direkter Kanülierung der Vorderkammer gemessen werden kann, zu entwickeln, um den Einfluss der CCT auf die Applanationstonometrie zu berücksichtigen, waren bislang widersprüchlich [2]. 
Tab. 1 Beurteilung der Vorderkammertiefe nach van Herick

van Herick- Verhältnis VKT:HH-Dicke Graduierung

Grad I VKT $=<1 / 4 \times$ HH-Dicke

Grad II VKT $=1 / 4 \times$ HH-Dicke

Grad III VKT $=1 / 4-1 / 2 \times$ HH-Dicke

Grad IV VKT $=$ HH-Dicke

VKT Vorderkammertiefe, HH-Dicke Hornhautdicke.

\section{Messung des Intraokulardruckes}

Der IOD sollte, wenn immer möglich, mittels Goldmann-Applanationstonometrie (GAT) gemessen werden. Sollten andere Messverfahren ([8]; z. B. dynamische Konturtonometrie, DCT) eingesetzt werden, dann lediglich in Ergänzung zur GAT.

Die „Non-contact-Air-Tonometrie“ ist aufgrund der nachgewiesenen großen Schwankungsbreite der Messungen [15] zum Management bei Patienten mit OHT oder Glaukom weniger geeignet.

\section{Perimetrie}

Definitionsgemäß ist das Gesichtsfeld bei Patienten mit OHT nicht beeinträchtigt.

Idealerweise erfolgt die Untersuchung als standardisierte, automatisierte, statische Perimetrie mit vollständiger Schwelleneingabelung (z. B. Humphrey 24-2, Octopus G1/G2).

Wie bei der Interpretation der Ergebnisse für Glaukompatienten muss auch bei Patienten mit OHT ein gewisser Lerneffekt für die ersten 2 bis 3 Untersuchungen bei der Interpretation der Befunde berücksichtigt werden (• Abb. 1, 2).

Es gibt Hinweise dafür, dass frühe Gesichtsfelddefekte mit alternativen Perimetrietechniken unter Umständen besser dargestellt werden können. Dazu zählen die Blau-Gelb-Perimetrie, die FDT-Technik (Frequenzverdopplungstechnik, „frequency doubling technology") oder das Heidelberg Edge-Perimeter („flicker-defined forms", FDF).

\section{Papillenbeurteilung}

Die subjektive Papillenbeurteilung sollte biomikroskopisch an der Spaltlampe
Infobox 2 Risikorechner

Der Risikorechner basiert auf den

Ergebnissen der Ocular Hypertension

Treatment Study (OHTS) und der European

Glaucoma Prevention Study (EGPS),

www.ohts.wustl.edu/risk/calculator.html

erfolgen, sodass eine ausreichende Vergrößerung zur detaillierten Beurteilung erreicht wird. Da eine stereoskopische Untersuchung die beste Beurteilung erlaubt, sollte die Basisuntersuchung in Mydriase erfolgen.

Die Beurteilung erfolgt analog der Beurteilung bei Glaukompatienten mit Augenmerk auf die Größe der Papille und der Exkavation sowie auf die „cup-todisc ratio“ („c/d-ratio") oder auch „rimto-disc“, die Randsaumverteilung (,ISNTrule") und das Vorliegen von Papillenrandblutungen (• Abb. 3).

\section{Papillen- und \\ Nervenfaserdokumentation}

Zusätzlich zur subjektiven, biomikroskopischen Papillenbeurteilung sollte eine objektive Dokumentation erfolgen. Dabei kann entweder die Papillenmorphologie oder die peripapilläre Nervenfaserschicht dokumentiert werden oder idealerweise beides.

Die Papillenmorphologie kann mittels Farbfotografie (• Abb.4), wenn immer möglich als Stereofarbfotografie, oder mit der Heidelberg-Retina-Tomographie (HRT) in vielen Fällen gut dokumentiert werden. Mit Einschränkungen ist dies mittlerweile auch bei einigen Herstellern mit der „Spectral-domain-optische-Kohärenztomographie" (SD-OCT, - Abb. 5) möglich.

Zur Darstellung der peripapillären Nervenfaserschicht (RNFL) stehen Scanning-Laser-Polarimeter (GDx-VCC oder -ECC) und SD-OCT zur Verfügung. Mit dem HRT ist die Messung der RNFL direkt nicht möglich, da lediglich eine Oberflächentopographie erfolgt. Das Gerät bedient sich jedoch eines Tricks, indem es eine Referenzebene $50 \mu \mathrm{m}$ unterhalb der Netzhautoberfläche auf Höhe des papillomakulären Bündels legt. Die Dicke von dieser Referenzebene bis zur Netzhautoberfläche wird als RNFL angenommen. Das HRT misst daher nicht die
Ophthalmologe $2011 \cdot 108: 1016-1022$

DOI 10.1007/s00347-011-2382-1

C) Springer-Verlag 2011

M. Töteberg-Harms · C. Hirn · J. Funk Empfehlungen notwendiger Kontrolluntersuchungen bei okulärer Hypertension

Zusammenfassung

Die okuläre Hypertension bezeichnet einen über die "Norm" (> 21 mmHg) erhöhten Intraokulardruck (IOD). Der Kammerwinkel ist definitionsgemäß offen, glaukomtypische Gesichtsfeldausfälle und Papillenveränderungen fehlen. Es handelt sich um Individuen, die nicht an einem Glaukom leiden, wohl aber ein erhöhtes Risiko haben, ein Glaukom zu entwickeln. Um glaukomtypische Gesichtsfeldausfälle und Papillenveränderungen auszuschließen, ist eine ausführliche "Glaukombasisdiagnostik" unabdingbar. Aufgrund des erhöhten Risikos, ein Glaukom zu entwickeln, sind feste Kontrollintervalle und eine standardisierte Untersuchung für das Followup ebenso zwingend erforderlich.

Schlüsselwörter

Okuläre Hypertension · Diagnose · Follow-up . Papillendokumentation · Augeninnendruck

\section{Recommendations for follow-up examinations in patients with ocular hypertension}

\section{Abstract}

Ocular hypertension (OHT) describes a condition with elevated intraocular pressure (IOP) above the norm ( $>21 \mathrm{mmHg}$ ). By definition the anterior chamber angle is open and there are neither signs of glaucomatous optic nerve damage nor visual field defects. Thus, for the diagnosis of $\mathrm{OHT}$ an extensive baseline examination is mandatory in order to rule out preexisting glaucomatous damage. Patients with ocular hypertension are at a higher risk of developing glaucoma. Therefore, periodical routine examinations with a standardized protocol for specific follow-up examinations are highly recommended.

Keywords

Ocular hypertension · Diagnosis · Follow-up . Optic nerve head imaging $\cdot$ Intraocular pressure 


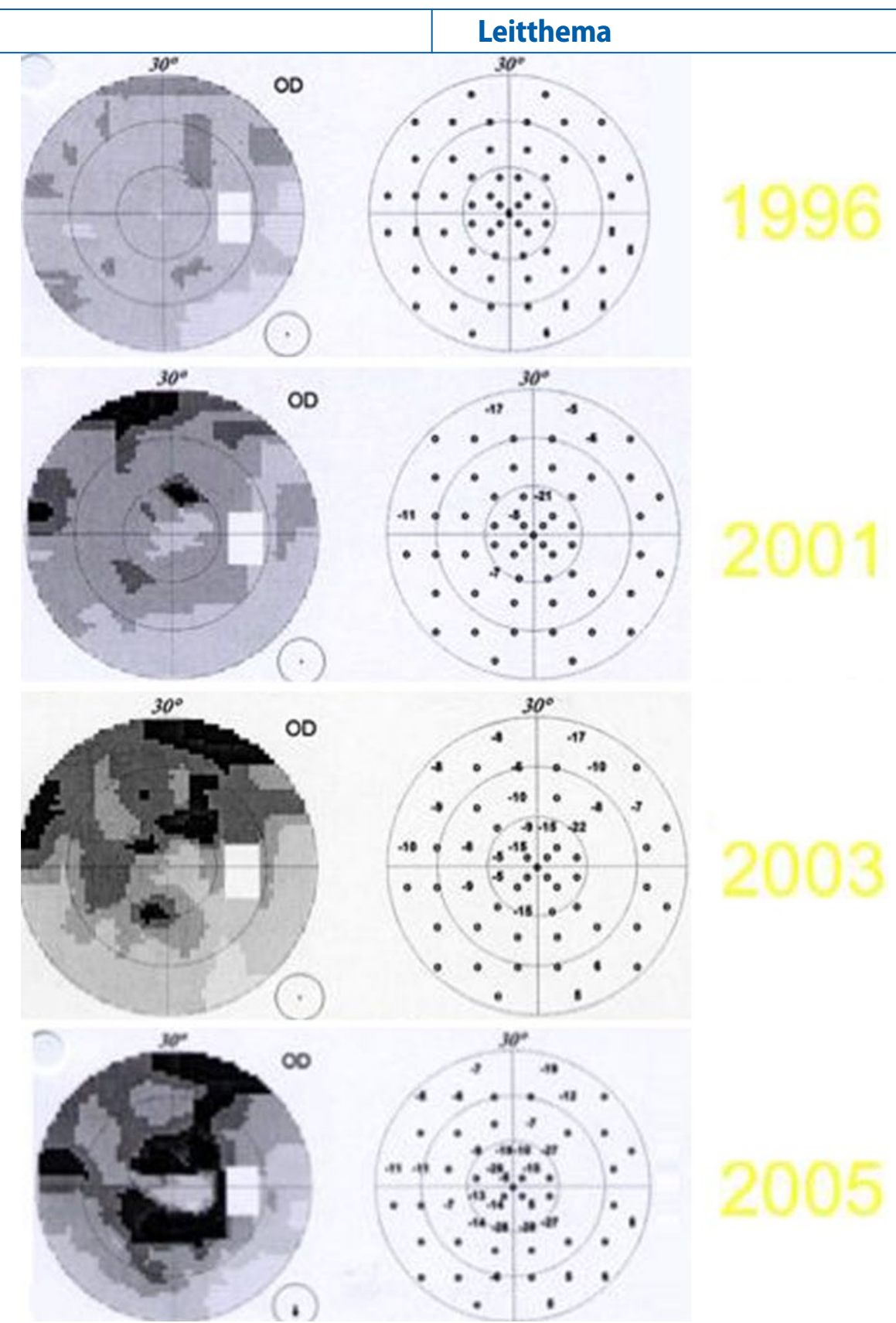

Abb. $2 \Delta$ Perimetrische Follow-up-Untersuchungen über 9 Jahre zeigen eine echte Progression zum Glaukom mit zunehmenden Gesichtsfelddefekten

Dicke der RNFL selbst, kann aber die Abnahme der RNFL mit der Zeit detektieren. Die Polarimetrie erlaubt eine topographische Bewertung der Vitalität der Nervenfaserschicht und das SD-OCT eine hochpräzise Messung der Nervenfaserschichtdicke bis auf wenige Mikrometer genau. Die diagnostische Aussagekraft dieser technisch gänzlich unterschiedlichen Verfahren ist in etwa gleichwertig, sodass im klinischen Alltag keiner Methode eindeutig der Vorzug gegeben werden muss. unkorrigierte Goldmann-Applanationsdruckwerte sein müssen, da die Hornhautdicke als eigenständiger Risikofaktor berücksichtigt wird.

\section{Follow-up-Untersuchungen}

Ziel der Verlaufsuntersuchungen bei OHT ist, eine Konversion in ein Glaukom möglichst frühzeitig zu erfassen. Routinemäßig müssen dafür aber nicht alle Untersuchungen der Basisdiagnostik wiederholt werden.

\section{Anamnese}

Änderungen der allgemeinen Medikation sollten regelmäßig erfragt werden.

\section{Spaltlampenuntersuchung und Gonioskopie}

Für Verlaufsuntersuchungen ist es ausreichend, die Vorderkammertiefe mit der Methode nach van Herick abzuschätzen. Eine routinemäßige Wiederholung der Gonioskopie ist nicht zwingend nötig.

\section{Pachymetrie}

Prinzipiell erfolgt die Pachymetrie einmalig bei der Basisdiagnostik. Wiederholt werden sollte die Pachymetrie bei einer zu erwartenden Veränderung des Befundes wie z. B. nach refraktiver Hornhautchirurgie.

\section{Messung des Intraokulardruckes}

Der IOD sollte routinemäßig bei jeder ophthalmologischen Konsultation gemessen werden.

\section{Perimetrie}

Basierend auf den Daten der OHTS und der European Glaucoma Prevention Study (EGPS), wurde ein Risikorechner entwickelt, der anhand mehrerer Parameter das relative 5-Jahres-Risiko für eine Konversion in ein Glaukom berechnet (• Infobox 2). Berücksichtigt werden das Alter des Patienten, der IOD, die vertikale „c/d-ratio“, die CCT und Gesichtsfeldparameter („pattern standard deviation“ oder „loss of variance“). Zu beachten ist, dass die eingegebenen Augendruckwerte
Es sollte darauf geachtet werden, dass für Verlaufsuntersuchungen stets ein Gerät des gleichen Herstellers und die gleiche Teststrategie verwendet werden, um Veränderungen sicher beurteilen zu können. Soweit möglich, sollten automatisierte Methoden zur Progressionsanalyse verwendet werden. 


\section{Papillenbeurteilung}

Im Verlauf sollte das Augenmerk auf die „c/ d-ratio“, die „ISNT-rule“ und das Vorliegen von Papillenrandblutungen gelegt werden.

\section{Papillen- und Nervenfaserdokumentation}

Für die Verlaufsdokumentation sollte stets das gleiche Verfahren angewendet werden, um Veränderungen möglichst früh erfassen zu können.

Bei Farbfotografien ist - abgesehen von der recht aufwendigen Planimetrie [5] - nur eine subjektive Beurteilung von Veränderungen möglich. GDx und SD-OCT ermöglichen einen Vergleich von Messparametern zu unterschiedlichen Zeitpunkten, HRT bietet 2 Arten automatisierter Progressionsanalyse.

Bei der Farbfotografie sowie implementiert in die Software des HRT besteht zusätzlich die Möglichkeit, Verlaufsbilder mit Grafikprogrammen übereinander zu legen und durch raschen Wechsel zwischen den Bildern eine Veränderung aufzudecken („Flickervergleich“). Die Bilder werden hierzu nach Größe und Winkel aneinander angeglichen.

\section{Untersuchungsintervalle}

Über die optimale Art der Dokumentation (Fotografie, HRT, GDx, OCT) sowie die optimale Anzahl und Frequenz der Verlaufsuntersuchungen, gibt es immer noch Uneinigkeit. Auch wenn einzelne Studien dies insbesondere für die Perimetrie bereits evaluiert haben [3]. Auch die European Glaucoma Society schlägt in ihren aktuellen Guidelines (3. Auflage) konkrete Intervalle vor (s. www.eugs.org).

Generell ist empfehlenswert, folgende Untersuchungen in Abständen von 6-12 Monaten durchzuführen:

- Anamnese,

- Spaltlampenuntersuchung,

- Messung des Intraokulardruckes,

- Perimetrie,

- biomikroskopische Papillenbeurteilung.
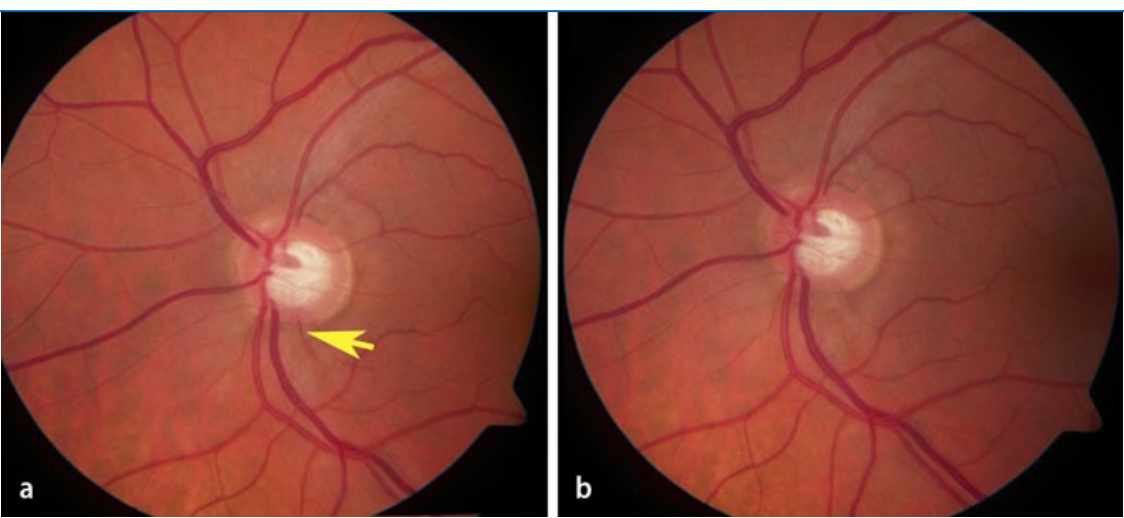

Abb. $3 \Delta$ a Die Papillenfarbfotografie zu unterschiedlichen Untersuchungszeitpunkten zeigt eine Papillenrandblutung bei der Basisuntersuchung bei 5:30 Uhr (gelber Pfeil), die leicht übersehen bzw. mit einem Gefäß verwechselt werden kann. b Die Papillenrandblutung ist bei der Dokumentation anlässlich der Follow-up-Untersuchung nicht mehr sichtbar, dafür fällt nun an dieser Stelle ein resultierender Nervenfaserbündeldefekt auf
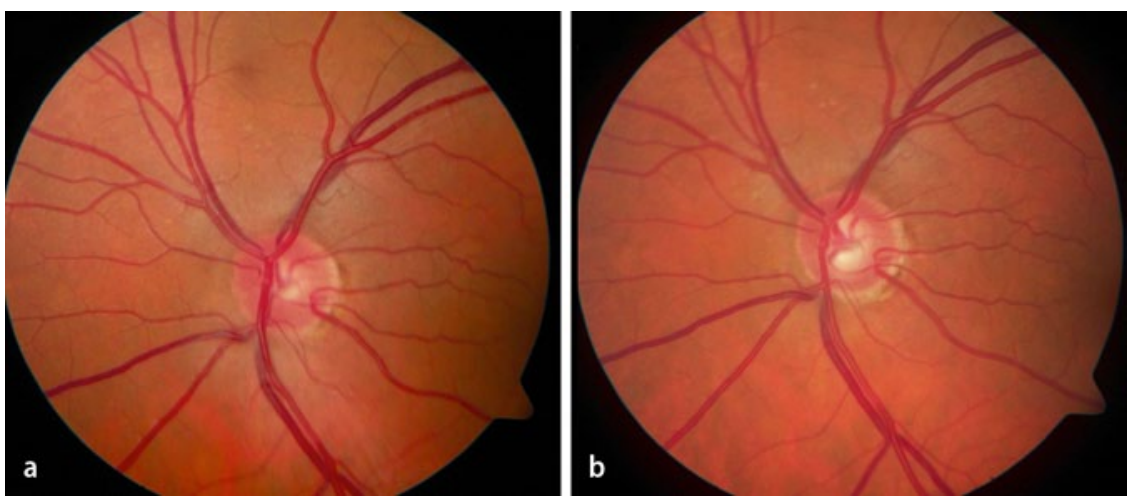

Abb. 4 \ Die Papillenfarbfotografie zu unterschiedlichen Untersuchungszeitpunkten zeigt im Vergleich zur Voraufnahme (a) eine Progression der Exkavation beim Follow-up (b)

Folgende Untersuchungen sollten in Abständen von 12-24 Monaten durchgeführt werden:

- objektive Papillendokumentation und

- Nervenfaserdokumentation

\section{Fazit für die Praxis}

\section{- Auch bei stabilen Befunden sollten} mindestens jährliche Kontrollintervalle beibehalten werden, da im Verlauf das höhere Alter des Patienten als eigenständiger Risikofaktor für die Entwicklung eines Glaukoms hinzukommt.

- Wesentliche Pfeiler der Verlaufskontrollen sind neben der Augendruckmessung die Perimetrie sowie die qualitative und quantitative Papillenbeurteilung und -dokumentation.

\section{Korrespondenzadresse \\ Dr. M. Töteberg-Harms

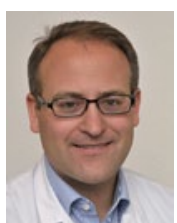 \\ Augenklinik, \\ UniversitätsSpital Zürich \\ Frauenklinikstr. 24, 8091 Zürich Schweiz \\ marc.toeteberg@usz.ch}

Interessenkonflikt. Der korrespondierende Autor gibt an, dass kein Interessenkonflikt besteht.

\section{Literatur}

1. Bankes JL, Perkins ES, Tsolakis S et al (1968) Bedford glaucoma survey. Br Med J 1:791-796

2. Brandt JD (2007) Central corneal thickness, tonometry, and glaucoma risk - a guide for the perplexed. Can J Ophthalmol 42:562-566

3. Chauhan BC, Garway-Heath DF, Goni FJ et al (2008) Practical recommendations for measuring rates of visual field change in glaucoma. $\mathrm{Br} \mathrm{J}$ Ophthalmol 92:569-573

4. Congdon NG, Spaeth GL, Augsburger J et al (1999) A proposed simple method for measurement in the anterior chamber angle: biometric gonioscopy. Ophthalmology 106:2161-2167 

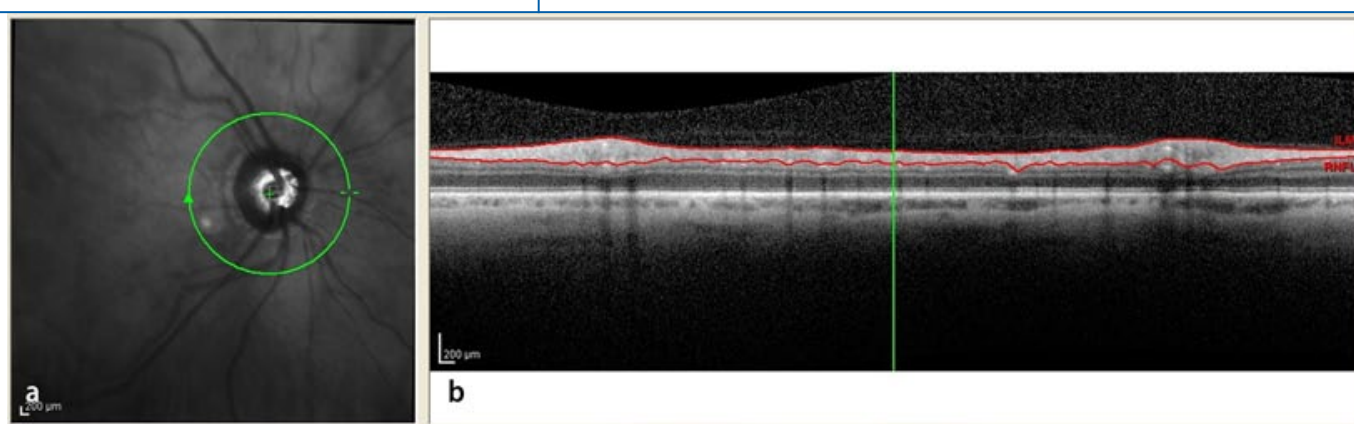

b

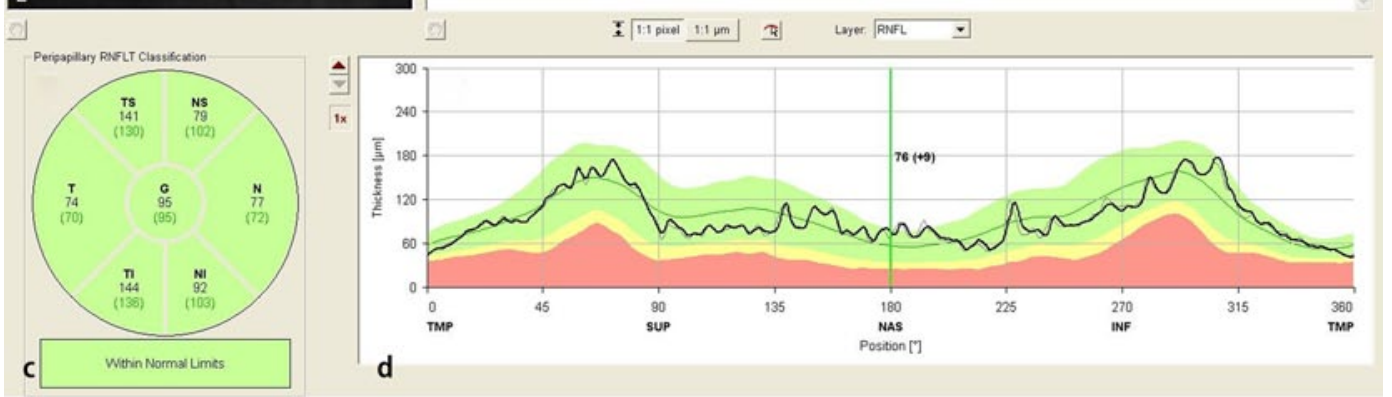

RNFL-Trend mit FoDi ${ }^{\mathrm{m}}$

SPECTRALIS叉 Tracking Laser Tomographie

Patient:

Geb.-Dat:

Geschlecht:

$\mathbf{R}$

Normierte RNFL-Dicke

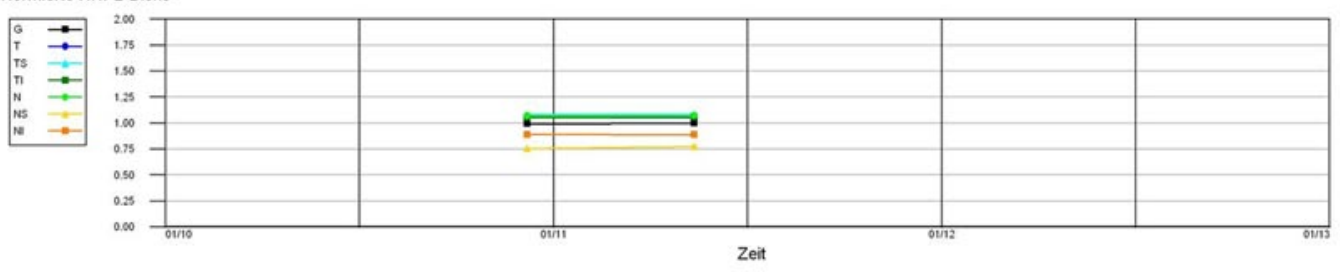

\begin{tabular}{|c|c|c|}
\hline Unters.-Datum & 07.Dez.2010 & 13.Mai.2011 \\
\hline RNFL-Dicke & & \\
\hline
\end{tabular}

Anderung zur

Referenz-
Aufnahme

e

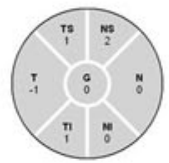

Abb. $5<$ RNFL-OCT (Heidelberg Spectralis ${ }^{\oplus}$ ). a Lage des peripapillären Scans im SLO-Bild, $\mathbf{b}$ automatische Erkennung der RNFL (rote Linien), c Dicke der RNFL in einzelnen Sektoren und global in Mikrometern und d als Profil. e Zusätzlich werden mittels eines Software-Tools (FoDi ${ }^{\oplus}$ ) einzelne Untersuchungen miteinander verglichen und die Progression ausgewertet
5. Jonas JB, Mardin CY, Grundler AE (1998) Comparison of measurements of neuroretinal rim area between confocal laser scanning tomography and planimetry of photographs. $\mathrm{Br} \mathrm{J}$ Ophthalmol 82:362-366

6. Kass MA, Heuer DK, Higginbotham EJ et al (2002) The Ocular Hypertension Treatment Study: a randomized trial determines that topical ocular hypotensive medication delays or prevents the onset of primary open-angle glaucoma. Arch Ophthalmol 120:701-713; discussion 829-730

7. Kitazawa Y, Horie T, Aoki S et al (1977) Untreated ocular hypertension. A long-term prospective study. Arch Ophthalmol 95:1180-1184

8. Kniestedt C, Punjabi O, Lin S et al (2008) Tonometry through the ages. Surv Ophthalmol 53:568591
9. Leibowitz HM, Krueger DE, Maunder LR et al (1980) The Framingham Eye Study monograph: an ophthalmological and epidemiological study of cataract, glaucoma, diabetic retinopathy, macular degeneration, and visual acuity in a general population of 2631 adults, 1973-1975. Surv Ophthalmol 24:335-610

10. Leske MC, Connell AM, Wu SY et al (1997) Distribution of intraocular pressure. The Barbados Eye Study. Arch Ophthalmol 115:1051-1057

11. Perkins ES (1973) The Bedford glaucoma survey. I. Long-term follow-up of borderline cases. $\mathrm{Br} J$ Ophthalmol 57:179-185

12. Pfeiffer N, Torri V, Miglior S et al (2007) Central corneal thickness in the European Glaucoma Prevention Study. Ophthalmology 114:454-459

13. Shaffer R (1962) Stereoscopic manual of gonioscopy. Mosby, St. Louis
14. Van Herick W, Shaffer RN, Schwartz A (1969) Estimation of width of angle of anterior chamber. Incidence and significance of the narrow angle. Am J Ophthalmol 68:626-629

15. Yucel AA, Sturmer J, Gloor B (1990) Comparison of tonometry with the Keeler air puff non-contact tonometer „Pulsair" and the Goldmann applanation tonometer. Klin Monatsbl Augenheilkd 197:329-334 